\title{
Acoustic Detection of Buried Objects in 3-D Fluid Saturated Porous Media: Numerical Modeling
}

\author{
Yan Qing Zeng, Student Member, IEEE, and Qing Huo Liu, Senior Member, IEEE
}

\begin{abstract}
Acoustic waves can be a viable tool for the detection and identification of land mines, unexplored ordnance (UXO), and other buried objects. Design of acoustic instruments and interpretation and processing of acoustic measurements call for accurate numerical models to simulate acoustic wave propagation in a heterogeneous soil with buried objects. Compared with the traditional seismic exploration, high attenuation is unfortunately ubiquitous for shallow surface acoustic measurements because of the loose soil and the fluid in its pore space. To adequately model such acoustic attenuation, we propose a comprehensive multidimensional finite-difference time-domain (FDTD) model to simulate the acoustic wave interactions with land mines and soils based on the Biot theory for poroelastic media. For the truncation of the computational domain, we use the perfectly matched layer (PML). The method is validated by comparison with analytical solutions. Unlike the pure elastic wave model, this efficient PML-FDTD model for poroelastic media incorporates the interactions of waves and the fluid-saturated pore space. Several typical land mine detection measurements are simulated to illustrate the application.
\end{abstract}

Index Terms-Acoustic waves, finite-difference time-domain (FDTD), landmine detection, perfectly matched layer, poroelasticity.

\section{INTRODUCTION}

$\mathbf{R}$ ECENTLY, acoustic methods have received considerable attention in the characterization of shallow objects. It has been shown that acoustic waves can be a viable tool for the detection and identification of land mines and unexplored ordnance (UXO) [1], [2] (also see some experimental papers in this special issue). The critical issue of acoustic wave coupling into the soil has been addressed by using low-frequency loudspeakers, laser, off-set mechanical source, and electric spark sources [2], [1]. These recent experimental efforts have shown promising results for acoustic landmine detection.

Design of acoustic instruments, interpretation and processing of acoustic measurements call for accurate numerical models to simulate acoustic wave propagation in a heterogeneous soil with buried objects. Conventional methods for acoustic modeling include the popular finite-difference time-domain (FDTD) solution of pure elastic media [3], [4]. Unfortunately, the pure elastic model cannot adequately incorporate the attenuation mechanism in its governing equations, although some approximate models are possible [5].

Manuscript received July 5, 2000; revised December 28, 2000. This work was supported by the Army Research Office under Grant DAAH04-96-1-0448 (Demining MURI).

The authors are with the Department of Electrical and Computer Engineering, Duke University, Durham, NC 27708 USA (qhliu@ee.duke.edu).

Publisher Item Identifier S 0196-2892(01)04835-5.
Compared with the traditional seismic exploration, high attenuation is unfortunately ubiquitous for shallow surface acoustic measurements because of the loose soil and the fluid in its pore space. To adequately model such acoustic attenuation, we propose a comprehensive model to simulate the acoustic wave interactions with land mines and soils based on the Biot theory [6]-[8] for poroelastic media. The FDTD method is then used to solve the Biot equations [8]. Similar to the velocity-stress finite-domain (FD) method [9], a velocity-strain, FD method is developed in a staggered grid for heterogeneous poroelastic media. In this method, Biot equations [8] are reformulated into first-order equations to arrive at a leap-frog system in a staggered grid both in time and space domains.

In order to simulate an unbounded medium, an absorbing boundary condition $(\mathrm{ABC})$ must be implemented to truncate the computational domain in numerical algorithms. Several ABCs have been developed for numerical simulation of wave propagation. Cerjan et al. [10] introduce a simple damping taper to the boundaries that attenuates outgoing waves. Since this lossy layer is not perfectly matched to the interior region, however, it requires a substantial number of layers for the taper to be effective. Clayton and Engquist [11] (CE) use the paraxial approximation to the wave equation to make the boundary transparent to outgoing waves. The $\mathrm{CE}$ ABC can lead to instability when the Poisson's ratio is greater than two [12]. Since Berenger [13] proposed the highly effective perfectly matched layer (PML) as an absorbing boundary condition for electromagnetic waves, it has been widely used for FD and finite-element methods. Chew and Liu [14] first proposed the PML for elastic waves in solids, and proved the zero reflections from PML to the regular elastic medium. Hastings et al. [15] have independently implemented the PML ABC for two-dimensional problems by using potentials. The PML has also been extended to model acoustic waves and electromagnetic waves in lossy media [16] and to cylindrical and spherical coordinates [17]. Recently, PML has been applied to the second-order Biot's equations for two-dimensional (2-D) fluid-saturated poroelastic media [18], which requires a complicated convolution. In this paper, we apply the PML to three-dimensional (3-D) using the first-order partial differential equation (PDE) system.

The difference between elastic model and poroelastic model is investigated by studying surface wave amplitude variation with offset (AVO) in three different types of soil: dry sand, fully water saturated sand and partly water saturated sand. The interaction of elastic wave with a plastic mine buried in dry sand is simulated. We also simulate two typical configurations for land mine detection, namely the excitation in soil [1] and the excitation in air [2]. The results show that the wave responses 
are significantly affected by the existence of a mine-like object through surface waves. After processing, the target can be detected by using surface acoustic measurements. The 2-D and 3-D poroelastic codes provide a useful tool for design and detection issues in acoustic characterization of buried objects.

\section{FORMULATION}

\section{A. Governing Equations}

The propagation of acoustic waves in fluid-saturated porous media is different from that in single phase elastic media. In addition to the regular $\mathrm{P}$ (compressional) waves and $\mathrm{S}$ (shear) waves in solid elastic media, a slow $\mathrm{P}$ wave resulting from the relative motion between the solid frame and pore fluid may be present in porous media. Thus, energy dissipation in porous media is different from that in solid elastic media. Based on continuum mechanics and macroscopic constitutive relationship, Biot [6]-[8] developed a theory of wave motion in a poroelastic solid saturated with a viscous compressible fluid. Biot's theory was confirmed by Burridge and Keller [19] based on the dynamic equations governing the behavior of medium on a microscopic scale. Plona [20] also confirmed Biot's theory through experiments.

In an isotropic, heterogeneous porous elastic medium, the parameters describing the physical properties of the medium are as follows:

$\mu \quad$ shear modulus of dry porous matrix;

$\lambda_{c} \quad$ Láme constant of saturated matrix;

$\phi \quad$ porosity;

$\eta \quad$ viscosity;

$\kappa \quad$ permeability;

$\rho \quad$ overall density of the saturated medium determined by $\rho_{f} \phi+(1-\phi) \rho_{s}$;

$\rho_{s} \quad$ density of solid material;

$\rho_{f} \quad$ density of fluid;

a tortuosity of the matrix;

$K_{s} \quad$ bulk modulus of the solid;

$K_{f} \quad$ bulk modulus of the fluid;

$K_{b} \quad$ bulk modulus of the dry porous frame.

The macroscopic displacements and strains are defined as $u_{i} \quad i$ th component of displacements of solid particle; $U_{i} \quad i$ th component of displacements of fluid particle;

$w_{i} \quad i$ th component of relative displacement, $w_{i}=\phi\left(U_{i}-u_{i}\right)$

$e_{i j} \quad i j$ component of strain tensor in porous medium;

$$
e_{i j}=\left[\left(\frac{\partial u_{j}}{\partial x_{i}}+\frac{\partial u_{i}}{\partial x_{j}}\right) / 2\right]
$$

e dilatation for the solid motion $e=\sum_{i=1,2,3} e_{i i}$;

$\xi \quad$ dilatation for the relative motion $\xi=-\sum_{i=1,2,3}$ $\left(\partial w_{i} / \partial x_{i}\right)$.

For a 3-D isotropic, heterogeneous and porous elastic medium, wave propagation is governed by Biot's equations [8]

$$
2 \sum_{j} \frac{\partial}{\partial x_{j}}\left(\mu e_{i j}\right)+\frac{\partial}{\partial x_{i}}\left(\lambda_{c} e-\alpha M \xi\right)=\frac{\partial^{2}}{\partial t^{2}}\left(\rho u_{i}+\rho_{f} w_{i}\right)
$$

$$
\frac{\partial}{\partial x_{i}}(\alpha M e-M \xi)=\frac{\partial^{2}}{\partial t^{2}}\left(\rho_{f} u_{i}+m w_{i}\right)+\frac{\eta}{\kappa} \frac{\partial w_{i}}{\partial t}
$$

where $m=a \rho_{f} / \phi$ and

$$
\begin{aligned}
M & =\frac{1}{\frac{\phi}{K_{f}}+\frac{(\alpha-\phi)}{K_{s}}} \\
\alpha & =1-\frac{K_{b}}{K_{s}} .
\end{aligned}
$$

Let $\mathbf{v}^{\mathbf{s}}=\partial \mathbf{u} / \partial t$ be the velocity of the solid particle and $\mathbf{v}^{\mathbf{f}}=\partial \mathbf{w} / \partial t$ be the velocity of the pore fluid relative to the solid frame. Then the second-order equations (1) and (2) can rearranged as the first-order equations

$$
\begin{aligned}
(m \rho & \left.-\rho_{f}^{2}\right) \frac{\partial v_{i}^{s}}{\partial t} \\
= & 2 m \sum_{j} \frac{\partial}{\partial x_{j}}\left(\mu e_{i j}\right)+m \frac{\partial}{\partial x_{i}}\left(\lambda_{c} e-\alpha M \xi\right) \\
& \quad-\rho_{f} \frac{\partial}{\partial x_{i}}(\alpha M e-M \xi)+\rho_{f} \frac{\eta}{\kappa} v_{i}^{f} \\
\left(m \rho-\rho_{f}^{2}\right) \frac{\partial v_{i}^{f}}{\partial t} & \\
= & \rho \frac{\partial}{\partial x_{i}}(\alpha M e-M \xi)-\rho \frac{\eta}{\kappa} v_{i}^{f}-2 \rho_{f} \sum_{j} \frac{\partial}{\partial x_{j}}\left(\mu e_{i j}\right) \\
& -\rho_{f} \frac{\partial}{\partial x_{i}}\left(\lambda_{c} e-\alpha M \xi\right)
\end{aligned}
$$

The time derivatives of strains $e_{i j}$ and $\xi$ can be expressed as

$$
\begin{aligned}
\frac{\partial e_{i j}}{\partial t} & =\left[\left(\partial v_{j}^{s} / \partial x_{i}+\partial v_{i}^{s} / \partial x_{j}\right) / 2\right] \\
\frac{\partial \xi}{\partial t} & =-\nabla \cdot \mathbf{v}^{\mathbf{f}} .
\end{aligned}
$$

In this work, an explicit second-order FD scheme is applied to convert (3)-(6) into a leap-frog system for the strain field $e_{i j}$ $\xi$ and velocity field $\mathbf{v}^{\mathbf{s}}$ and $\mathbf{v}^{\mathbf{f}}$. With proper absorbing boundary conditions, these equations can be solved numerically for the wave field in an unbounded medium.

Equations (3)-(6) predict the existence of three different waves in fluid-saturated poroelastic media: a shear wave and two compressional waves with a faster and a slower propagation velocities [6]-[8].

\section{B. Note for Pure Fluid and Solid}

Biot's theory is more general than the elastodynamic equations, and includes the latter as a special case. Therefore, equations (3) and (4) may also be used to describe the response of single-phase elastic media as well as a single-phase fluid. For a single-phase solid, we simply let porosity $\phi \rightarrow 0$ and the initial condition for the relative motion equal to zero. On the other hand, a single-phase fluid can be modeled by setting $K_{f}=K_{s}$ and $\mu=0$ explicitly in these equations. Given the zero initial relative motion, equation (4) will guarantee there is no relative motion for all time. Therefore, there is no need to introduce special treatment even if some regions of the model have pure fluid and solid. 


\section{Equations for the PML Absorbing Boundary Condition}

In this paper, the PML is used to truncate the unbounded medium to absorb all outgoing waves. This artificial absorptive medium is introduced in the regular medium by modifying equations (3)-(6) with complex coordinates [14], [17]. In the frequency domain with $e^{-i \omega t}$ convention, a complex coordinate variable is chosen as

$$
\begin{aligned}
& \widetilde{x_{j}}=\int_{0}^{x_{j}} e_{j}\left(x_{j}{ }^{\prime}\right) d x_{j}{ }^{\prime} \\
& e_{j}=a_{j}+i \frac{\omega_{j}}{\omega} \quad(j=1,2,3)
\end{aligned}
$$

where $a_{j} \geq 1$ is a scaling factor, and $\omega_{x_{j}} \geq 0$ is an attenuation factor. The operator $\partial / \partial x_{j}$ can be expressed in terms of the regular coordinate

$$
\frac{\partial}{\partial \widetilde{x_{j}}}=\frac{1}{e_{j}} \frac{\partial}{\partial x_{j}} .
$$

The PML formulation is to replace $x_{j}$ in (4)-(6) by the corresponding complex coordinate $\widetilde{x_{j}}$. In a regular non-PML region, $a_{j}=1$ and $\omega_{j}=0$. In order to simplify PML equations, the field variables are split as the following:

$$
\begin{aligned}
& v_{j}^{s}=\sum_{k=1}^{3} v_{j}^{s(k)} \\
& v_{j}^{f}=\sum_{k=1}^{3} v_{j}^{f(k)}
\end{aligned}
$$

where $v_{i}^{s(k)}$ and $v_{i}^{f(k)}$ represent the split field variables containing space derivative $\partial / \partial x_{k}$ only . For example, (3) for $i=$ 1 can be split into the following three equations:

$$
\begin{aligned}
\left(m \rho-\rho_{f}^{2}\right) \frac{\partial v_{1}^{s(1)}}{\partial t}= & 2 m \frac{\partial}{\partial x_{1}}\left(\mu e_{11}\right)+m \frac{\partial}{\partial x_{1}}\left(\lambda_{c} e-\alpha M \xi\right) \\
& -\rho_{f} \frac{\partial}{\partial x_{1}}(\alpha M e-M \xi) \\
\left(m \rho-\rho_{f}^{2}\right) \frac{\partial v_{1}^{s(2)}}{\partial t} & =2 m \frac{\partial}{\partial x_{2}}\left(\mu e_{12}\right)+\rho_{f} \frac{\eta}{\kappa} v_{1}^{f} \\
\left(m \rho-\rho_{f}^{2}\right) \frac{\partial v_{1}^{s(3)}}{\partial t} & =2 m \frac{\partial}{\partial x_{3}}\left(\mu e_{13}\right) .
\end{aligned}
$$

The diagonal strain components $e_{j j}$ need not be split. However, other strain components have to be split as $e_{j l}=\sum_{k=1}^{3} e_{j l}^{(k)}$ for $j \neq l$, and $\xi=\sum_{k=1}^{3} \xi^{(k)}$, for example

$$
\begin{aligned}
\frac{\partial e_{j j}}{\partial t} & =\frac{\partial v_{j}^{s}}{\partial x_{j}} \\
e_{12} & =e_{12}^{(1)}+e_{12}^{(2)} \\
\frac{\partial e_{12}^{(1)}}{\partial t} & =\frac{1}{2} \frac{\partial v_{2}^{s}}{\partial x_{1}} \\
\frac{\partial e_{12}^{(2)}}{\partial t} & =\frac{1}{2} \frac{\partial v_{1}^{s}}{\partial x_{2}} \\
\xi & =\xi^{(1)}+\xi^{(2)}+\xi^{(3)} \\
\frac{\partial \xi^{(1)}}{\partial t} & =-\frac{\partial v_{1}^{f}}{\partial x_{1}}
\end{aligned}
$$

$$
\begin{aligned}
& \frac{\partial \xi^{(2)}}{\partial t}=-\frac{\partial v_{2}^{f}}{\partial x_{2}} \\
& \frac{\partial \xi^{(3)}}{\partial t}=-\frac{\partial v_{3}^{f}}{\partial x_{3}} .
\end{aligned}
$$

In frequency domain PML formulation, after $x_{j}$ is replaced by $\widetilde{x_{j}}$, equations for $v_{1}^{s}$, for example, can be rewritten as

$$
\begin{aligned}
(m \rho & \left.-\rho_{f}^{2}\right)(-i \omega)\left(1+i \frac{\omega_{1}}{\omega}\right) v_{1}^{s(1)} \\
= & 2 m \frac{\partial}{\partial x_{1}}\left(\mu e_{11}\right)+m \frac{\partial}{\partial x_{1}}\left(\lambda_{c} e-\alpha M \xi\right) \\
& \quad-\rho_{f} \frac{\partial}{\partial x_{1}}(\alpha M e-M \xi) \\
\left(m \rho-\rho_{f}^{2}\right)(-i \omega)\left(1+i \frac{\omega_{2}}{\omega}\right) v_{1}^{s(2)} & \\
= & 2 m \frac{\partial}{\partial x_{2}}\left(\mu e_{12}\right)+\left(1+i \frac{\omega_{2}}{\omega}\right) \rho_{f} \frac{\eta}{\kappa} v_{1}^{f} \\
\left(m \rho-\rho_{f}^{2}\right)(-i \omega)\left(1+i \frac{\omega_{3}}{\omega}\right) v_{1}^{s(3)} & \\
= & 2 m \frac{\partial}{\partial x_{3}}\left(\mu e_{13}\right) .
\end{aligned}
$$

By taking an inverse Fourier transform (FT), the above equations yield the time-domain PML equations

$$
\begin{aligned}
& \left(m \rho-\rho_{f}^{2}\right)\left(\frac{\partial v_{1}^{s(1)}}{\partial t}+\omega_{1} v_{1}^{s(1)}\right) \\
& =2 m \frac{\partial}{\partial x_{1}}\left(\mu e_{11}\right)+m \frac{\partial}{\partial x_{1}}\left(\lambda_{c} e-\alpha M \xi\right) \\
& -\rho_{f} \frac{\partial}{\partial x_{1}}(\alpha M e-M \xi) \\
& \left(m \rho-\rho_{f}^{2}\right)\left(\frac{\partial v_{1}^{s(2)}}{\partial t}+\omega_{2} v_{1}^{s(2)}\right) \\
& =2 m \frac{\partial}{\partial x_{2}}\left(\mu e_{12}\right)+\rho_{f} \frac{\eta}{\kappa}\left(v_{1}^{f}+\omega_{2} \int_{-\infty}^{t} v_{1}^{f} d \tau\right) \\
& \left(m \rho-\rho_{f}^{2}\right)\left(\frac{\partial v_{1}^{s(3)}}{\partial t}+\omega_{3} v_{1}^{s(3)}\right) \\
& =2 m \frac{\partial}{\partial x_{3}}\left(\mu e_{13}\right) \\
& \frac{\partial e_{11}}{\partial t}=\frac{\partial v_{1}}{\partial x_{1}}-\omega_{1} e_{11} \\
& \frac{\partial e_{12}^{(1)}}{\partial t}=\frac{1}{2} \frac{\partial v_{2}^{s}}{\partial x_{1}}-\omega_{1} e_{12}^{(1)} \\
& \frac{\partial e_{12}^{(2)}}{\partial t}=\frac{1}{2} \frac{\partial v_{1}^{s}}{\partial x_{2}}-\omega_{2} e_{12}^{(2)} \\
& \frac{\partial \xi^{(1)}}{\partial t}=-\frac{\partial v_{1}^{f}}{\partial x_{1}}-\omega_{1} \xi^{(1)} \\
& \frac{\partial \xi^{(2)}}{\partial t}=-\frac{\partial v_{2}^{f}}{\partial x_{2}}-\omega_{2} \xi^{(2)} \\
& \frac{\partial \xi^{(3)}}{\partial t}=-\frac{\partial v_{3}^{f}}{\partial x_{3}}-\omega_{3} \xi^{(3)}
\end{aligned}
$$




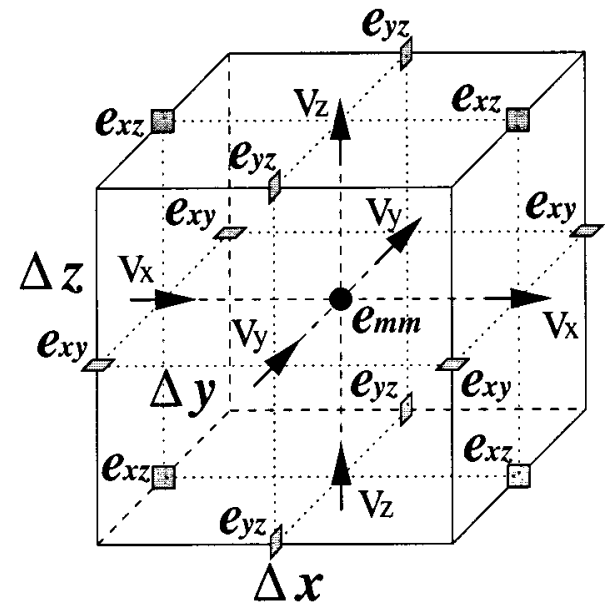

Fig. 1. Relative locations of field components in a unit cell of staggered grid.

Similar equations can be obtained in the same way for other components $v_{2}^{s}, v_{3}^{s}, v_{1}^{f}, v_{2}^{f}, v_{3}^{f}$, and $e_{13}, e_{23}$. Within the PML region, (3) and (4) are split into 18 equations for 3-D. Equation (5) is split into nine equations, and (6) is split into three equations. So the total number of the equations is 30 for the PML region, compared to 13 for the regular interior region. Thus, the memory requirement within the PML region is about two and half times that required by a regular medium for 3-D problems. This extra memory requirement in PML region is offset by the effectiveness of PML in absorbing the outgoing waves.

\section{FD Implementation}

The governing equations for the PML absorbing boundary condition, such as (10)-(18), are first-order partial differential equations for particle velocity and strain. They can be solved with different numerical methods. For the evaluation of seismic and acoustic responses, the FD method is widely used because of its flexibility and simplicity. Here, we use the explicit secondorder FD method with a staggered grid in both spatial and temporal domains.

To implement a 3-D FD method to the PML equations, the material parameters and unknown field components are discretized on a regular 3-D grid at the intervals $\Delta x_{1}, \Delta x_{2}$ and $\Delta x_{3}$. The time domain is also discretized with time step $\Delta t$. For the FD implementation of Biot's equations on a staggered grid in Fig. 1, the velocity field components are located at the cell's face centers, while material parameters and normal strains are located at the center of the cell and shear strains are located at the six edge centers. The strain field is computed at $n \Delta t$ and velocity field is computed at $(n+1 / 2) \Delta t$. This staggered grid is similar to that for elastic waves in a solid [4], [22].

With this discretization, a leap-frog time-stepping system can be obtained. In order to simplify the layout of the formulas, the governing equations with PML boundary condition can be generalized as the first-order differential equation. For example, (11), (13), and (16) can be rewritten as

$$
\begin{aligned}
\frac{\partial v_{1}^{s(2)}}{\partial t}+c_{0} v_{1}^{s(2)}= & c_{1} \int_{-\infty}^{t} v_{1}^{f} d \tau \\
& +c_{2}\left[2 m \frac{\partial}{\partial x_{2}}\left(\mu e_{12}\right)+\rho_{f} \frac{\eta}{\kappa}\left(v_{1}^{f}\right)\right]
\end{aligned}
$$

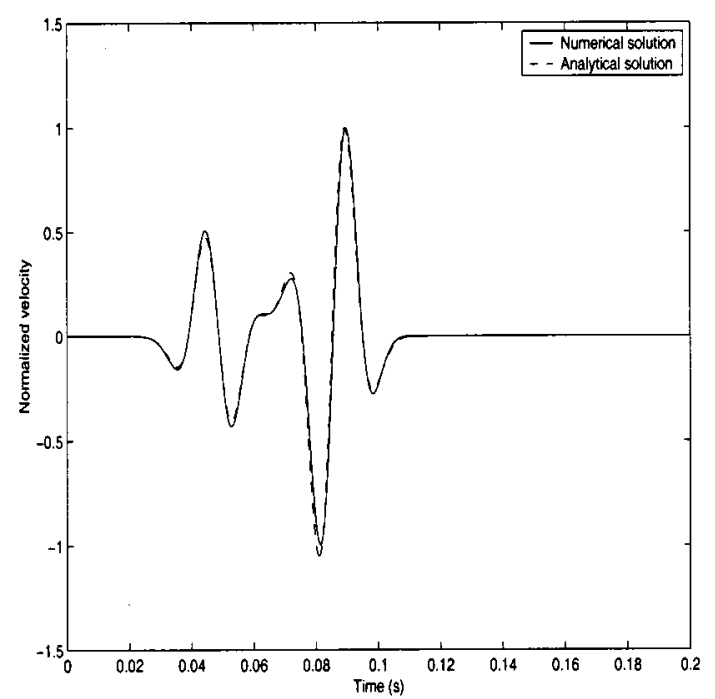

Fig. 2. Comparison of analytical and numerical solutions for the vertical velocity component in a homogeneous solid.

$$
\begin{aligned}
\frac{\partial e_{11}}{\partial t}+\omega_{1} e_{11} & =\frac{\partial v_{2}^{s}}{\partial x_{1}} \\
\frac{\partial \xi^{1}}{\partial t}+\omega_{1} \xi^{1} & =-\frac{\partial v_{1}^{f}}{\partial x_{1}}
\end{aligned}
$$

where $c_{0}, c_{1}$, and $c_{2}$ space-dependent coefficients. Then the time-stepping equations can be written as

$$
\begin{gathered}
v_{1}^{s(2)}\left[j_{1}, j_{2}, j_{3},\left(n+\frac{1}{2}\right)\right] \\
=f_{1} v_{1}^{s(2)}\left[j_{1}, j_{2}, j_{3},\left(n-\frac{1}{2}\right)\right]+f_{2} R_{1} \\
e_{11}\left[j_{1}, j_{2}, j_{3},(n+1)\right] \\
=g_{1} e_{11}\left[j_{1}, j_{2}, j_{3}, n\right]+g_{2} R_{2} \\
\xi^{1}\left[j_{1}, j_{2}, j_{3},(n+1)\right] \\
=g_{1} \xi^{1}\left[j_{1}, j_{2}, j_{3}, n\right]-g_{2} R_{3} \\
f_{1}=\frac{c_{0} / 2-1 / \Delta t}{1 / \Delta t+c_{0} / 2} \\
f_{2}=\frac{1}{1 / \Delta t+c_{0} / 2} \\
g_{1}=\frac{\omega_{1} / 2-1 / \Delta t}{1 / \Delta t+\omega_{1} / 2} \\
g_{2}=\frac{1}{1 / \Delta t+\omega_{1} / 2}
\end{gathered}
$$

where $R_{1}, R_{2}$, and $R_{3}$ are right-hand sides of equations (19), (20), and (21), respectively. It should be noted that the material parameters in the above equations must be properly averaged in order to arrive at a higher accuracy [4]. In order to save computer storage, the computational domain is divided into a PML region and an interior region. The absorption of outgoing waves is achieved by the PML region, which consists of several cells of PML materials with a quadratically tapered $\omega_{j}$ profile to in- 


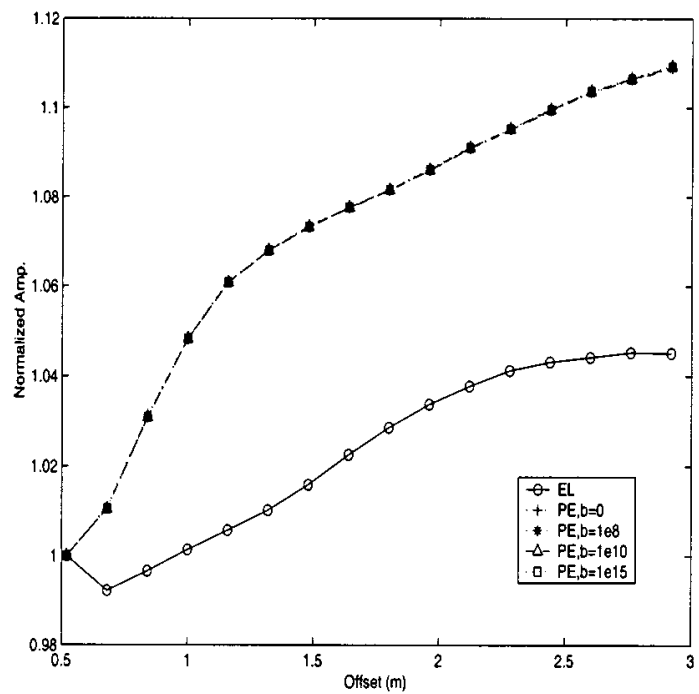

Fig. 3. Comparison between the elastic and poroelastic model for dry sand.

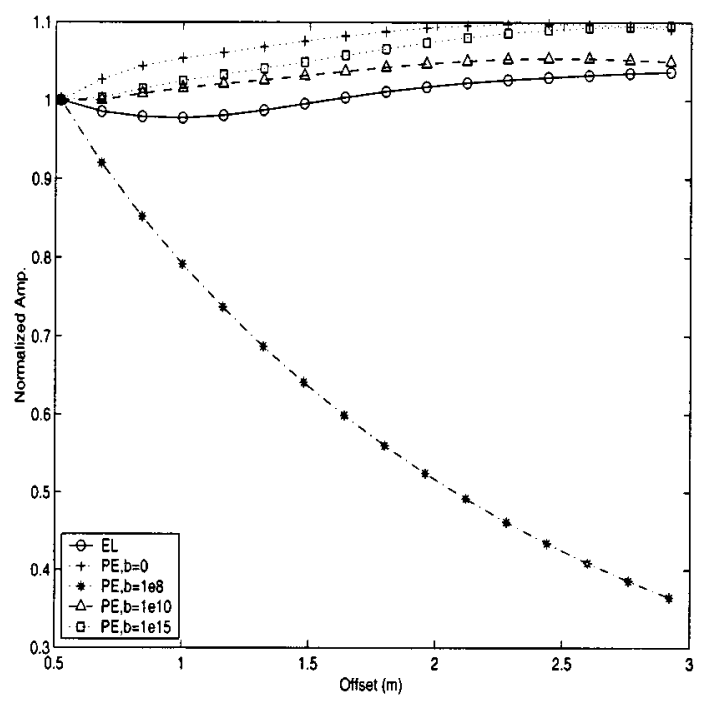

Fig. 4. Comparison between the elastic and poroelastic model for fully water-saturated sand.

crease the attenuation toward the outer boundary. In this paper, $\omega_{j}$ of the PML region is chosen as

$$
\omega_{j}(j)=\frac{(M-1 / 2-j)^{2}}{(M-1 / 2)^{2}} \omega_{j, \max }
$$

where $\omega_{j, \max }$ is the value at the center of the cell at the outermost boundary. At the outer boundary, the velocity components are forced to be zero. For convenience, $\omega_{j, \max }$ can be expressed in terms of dominant frequency and normalized coefficient. Then (25) becomes

$$
\omega_{j}= \begin{cases}2 \pi a_{0} f_{0}\left(l_{x_{j}} / L_{\mathrm{PML}}\right)^{2}, & \text { inside PML } \\ 0, & \text { outside PML }\end{cases}
$$

where

$f_{0} \quad$ dominant frequency of the source;

$L_{\text {PML }}$ thickness of the PML region;

$l_{x_{j}} \quad$ distance from the interface between the interior region and PML region.

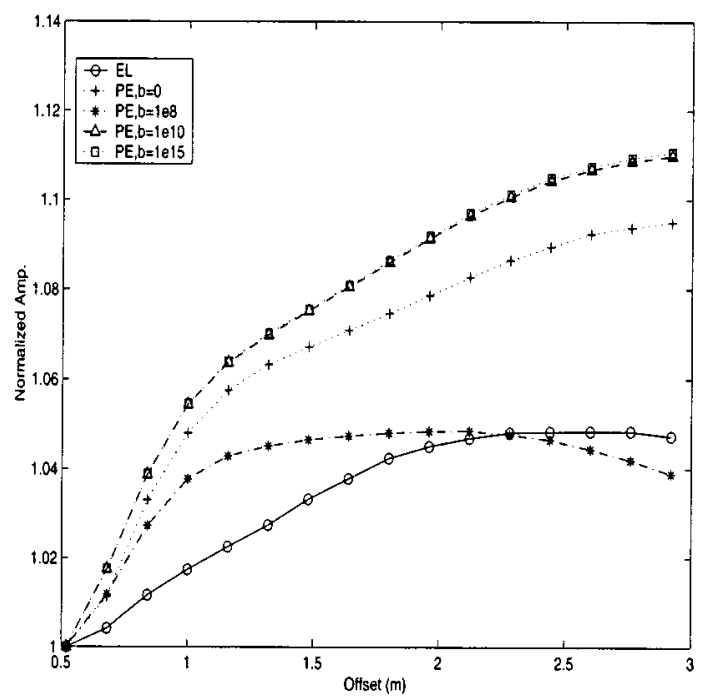

Fig. 5. Comparison between the elastic and poroelastic model for partly water-saturated sand.

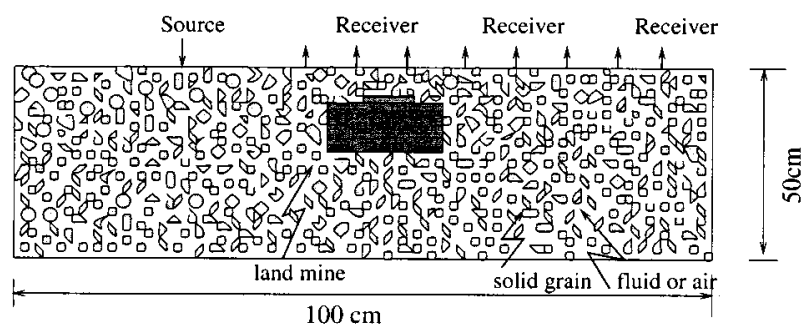

Top View of the Model

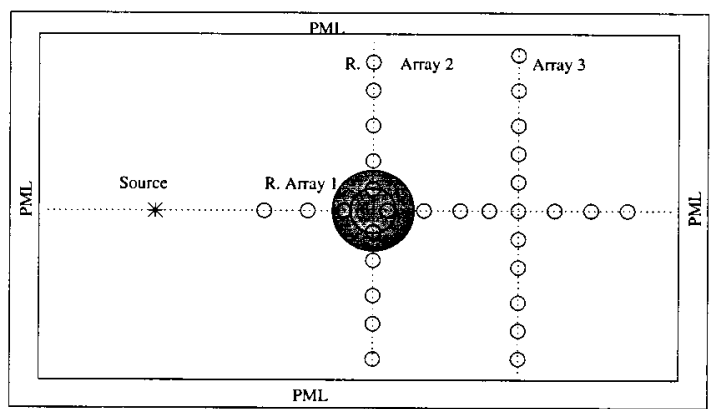

Fig. 6. Geometry of a buried land mine model.

\section{NUMERICAL RESULTS}

In the following numerical simulations, the source energy is partitioned linearly between the solid and the fluid phases with factors

$$
\begin{aligned}
& W_{f}=\phi \\
& W_{s}=(1-\phi) \\
& W_{r}=\phi\left|W_{f}-W_{s}\right|
\end{aligned}
$$

where

$W_{f} \quad$ weighting factor for the fluid motion;

$W_{s} \quad$ solid motion;

$W_{r} \quad$ relative motion between solid frame and pore fluid. 
(a)

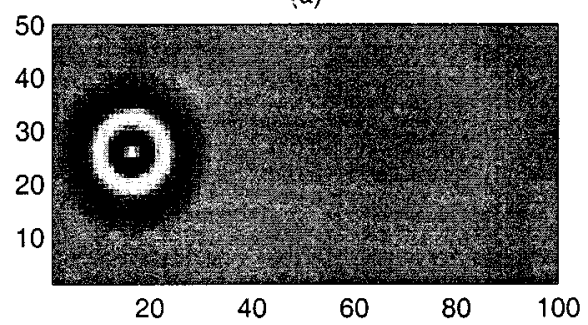

(c)

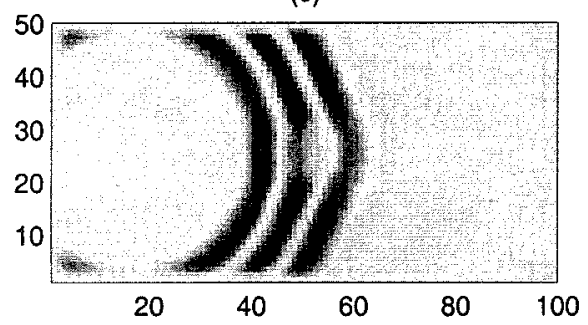

(b)

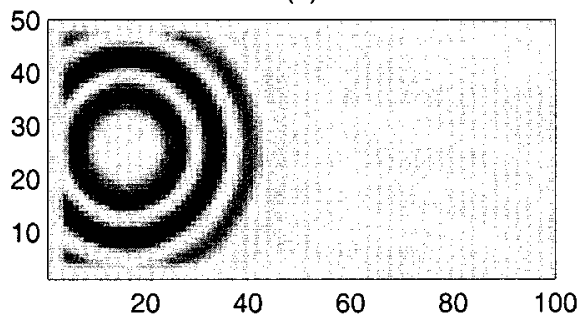

(d)

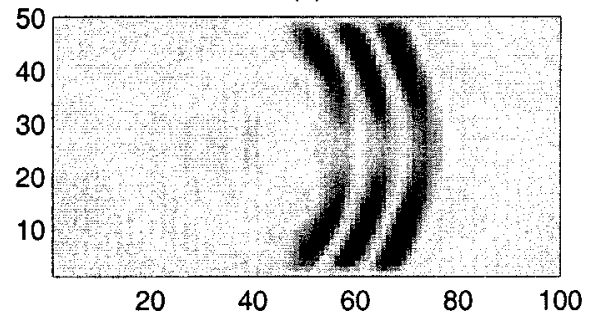

Fig. 7. Snapshots at (a) $2.3 \mathrm{~ms}$, (b) $4.0 \mathrm{~ms}$, (c) $6.0 \mathrm{~ms}$, and (d) $7.8 \mathrm{~ms}$. A dipole source is on the free surface.

\section{A. Validation of Numerical Results}

An analytical solution for the particle velocity field in a homogeneous, fluid-saturated poroelastic medium subject to a point source in 3-D space or a line source in 2-D space can be obtained in a closed form via potential functions [9]. In a poroelastic medium with an ideal nonviscous fluid, a purely dilatational source will only excite $\mathrm{P}$ waves given by scalar potentials

$$
\begin{aligned}
\psi_{s}(r, t) & =\frac{\alpha s\left(t-\frac{r}{V_{f}}\right)+\beta s\left(t-\frac{r}{V_{s}}\right)}{4 \pi r} \\
\psi_{f}(r, t) & =\frac{\alpha A_{f} s\left(t-\frac{r}{V_{f}}\right)+\beta A_{s} s\left(t-\frac{r}{V_{s}}\right)}{4 \pi r}
\end{aligned}
$$

where

$r \quad$ distance;

$s(t) \quad$ source time function;

$A_{f}$ and $A_{s}$ ratios between the solid and fluid motion for the fast P-wave and the slow P-wave, and the coefficients;

$\alpha$ and $\beta \quad$ determined by the regularity conditions;

$V_{f}$ and $V_{s}$ velocities of the fast $\mathrm{P}$-wave and the slow $\mathrm{P}$-wave, respectively.

The displacement field in the solid and fluid is then written respectively as $\mathbf{u}=\nabla \psi_{s}$ and $\mathbf{U}=\nabla \psi_{f}$.

The validation of the numerical method can be done by comparing the numerical results with the above analytical solution. A homogeneous model of sand saturated with water is considered. A P-wave point source of the first derivative Gaussian time function with $f_{0}=40 \mathrm{~Hz}$ is located at $(0,0,0)$. Then the solution at $(30 \mathrm{~m}, 30 \mathrm{~m}, 5 \mathrm{~m})$ is calculated. The numerical result and analytical solution in Fig. 2 have an excellent agreement.

\section{B. Comparison Between Elastic and Poroelastic Models}

In the conventional elastic model, a single-phase medium is considered. There is no energy dissipation in such an elastic medium. In poroelastic model, however, a multiphase medium

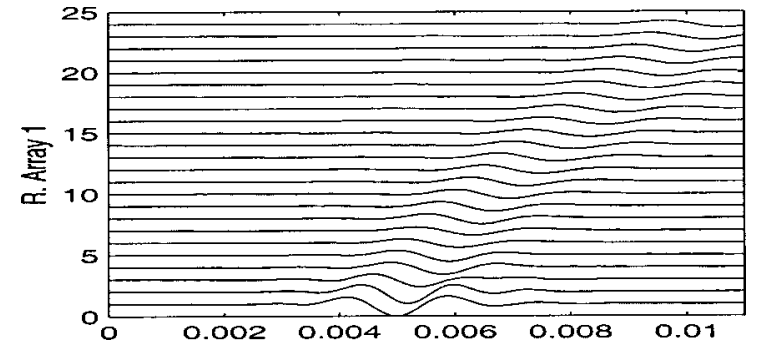

(a)

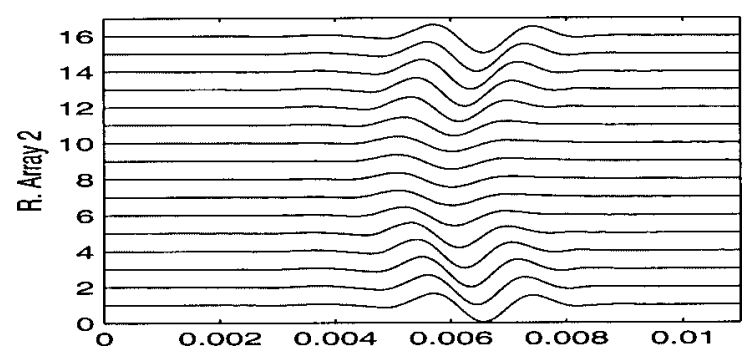

(b)

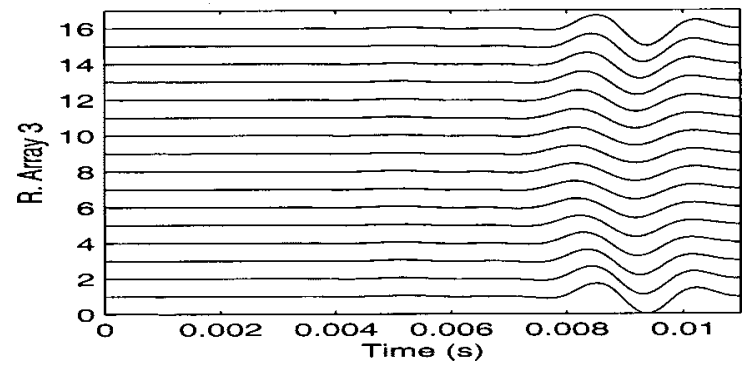

(c)

Fig. 8. Waveforms of vertical velocity of solid frame at receiver (a) array 1, (b) array 2, (c) array 3. A dipole source is on the free surface.

(usually solid frame and pore fluid) is considered. The in-phase motion between solid and fluid leads to the regular P-wave and $\mathrm{S}$-wave, and the out-phase motion between solid and fluid leads to the slow P wave. Because the fluid is viscous, there is energy dissipation between the solid frame and the pore fluid. In Biot's theory, the ratio of viscosity and permeability $(b=\eta / \kappa)$ 
(a)

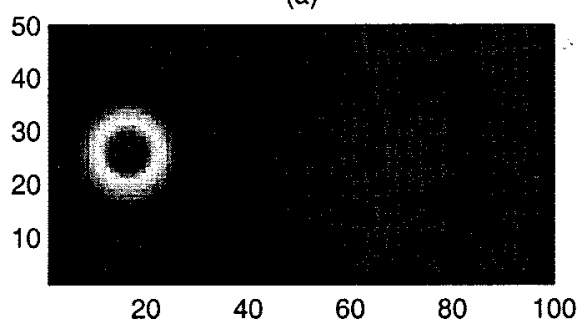

(c)

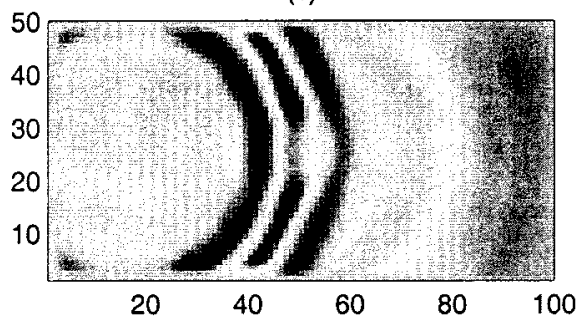

(b)

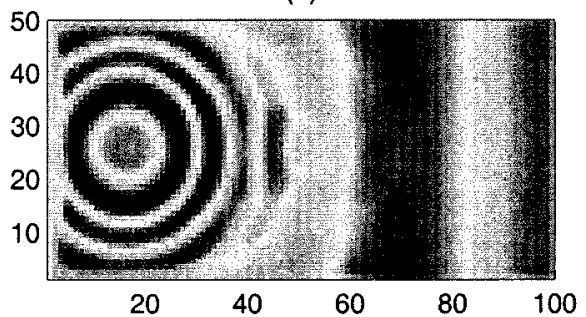

(d)

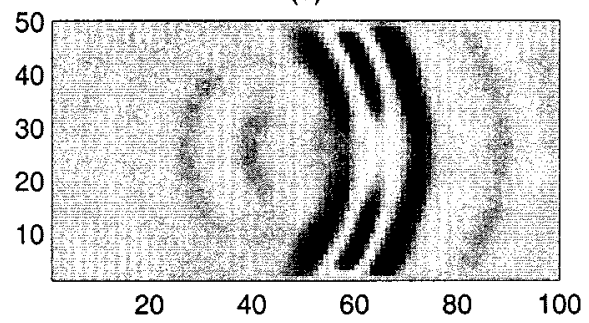

Fig. 9. Snapshots at (a) $2.3 \mathrm{~ms}$, (b) $4.0 \mathrm{~ms}$, (c) $6.0 \mathrm{~ms}$, and (d) $7.8 \mathrm{~ms}$. A monopole source is in the air.

determines this dissipation, as shown in (3) and (4). The difference between an elastic model and a poroelastic model is investigated here by studying the effect of $b$ on AVO response in three different ground media: dry sand, fully water saturated sand, and partly water saturated sand (70\% air and $30 \%$ water). For a half-space problem, since the source and receivers are usually on the free surface and the surface wave is the dominant signal at low frequencies, only the surface wave is investigated here. In this case, we model the source as a vertical dipole right on the ground surface and displaced horizontally away from the buried object, a scenario similar to the source in [1].

Figs. 3-5 show the effects of $b$ on the surface wave AVO for dry sand, fully water saturated sand, and partly water-saturated sand models, respectively. For the dry sand, the difference between elastic and poroelastic models is obvious, but $b$ has little effect on AVO. For the fully water saturated sand model, $b$ has a significant effect on AVO. When $b$ is very large, the AVO response is very close to that of the elastic model. For the partly water saturated sand model, the difference between the elastic and poroelastic model is obvious, and $b$ also has a significant effect on AVO.

\section{Acoustic Land Mine Detection Systems}

This method is used to model a plastic antipersonnel mine that is difficult to detect with the more conventional electromagnetic induction sensors (i.e., metal detectors). The geometry of the model of a buried mine is shown in Fig. 6. The source can be either on the surface or in the air. There are three arrays of receivers on the surface to pick up the signal of vertical velocity.

The parameters for the plastic mine are chosen as $\rho=1200$ $\mathrm{kg} / \mathrm{m}^{3}, \mathrm{~S}$-wave velocity $v_{s h}=1100 \mathrm{~m} / \mathrm{s}$ and P-wave velocity $v_{p}=2700 \mathrm{~m} / \mathrm{s}$. The material in the ground may be considered as a two-phase composite material consisting of granular solid and pore fluid. The nature of this composite varies with environment, geographic location, and with depth below the surface of ground. In this model, the soil is chosen as dry sand and the porosity is 0.35 . The fast $\mathrm{P}$ wave velocity is $250 \mathrm{~m} / \mathrm{s}$ and $\mathrm{S}$ wave velocity is $87 \mathrm{~m} / \mathrm{s}$.

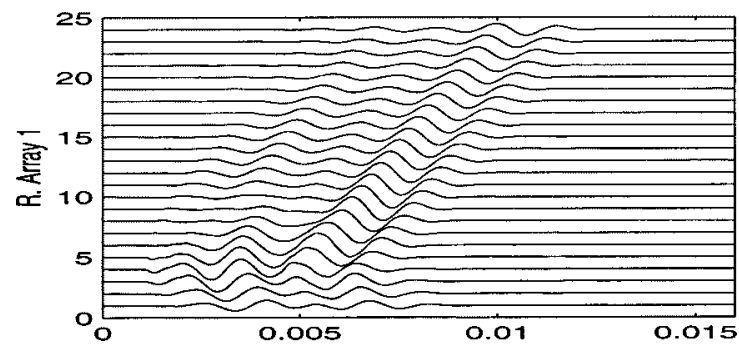

(a)

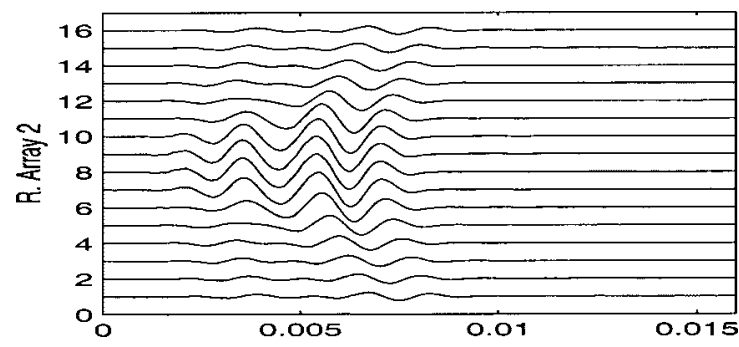

(b)

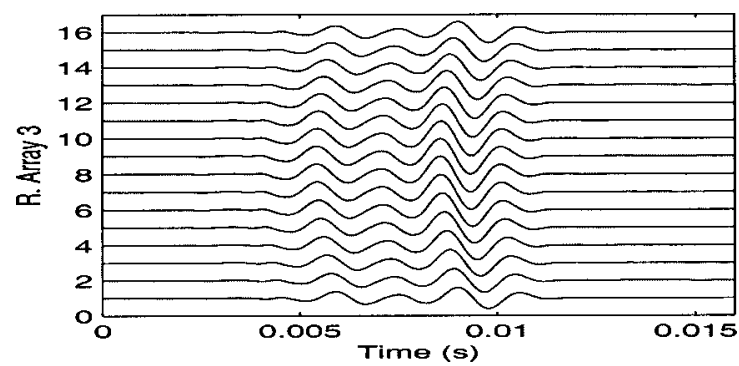

(c)

Fig. 10. Waveforms of scattered field of solid frame at receiver (a) array 1, (b) array 2, (c) array 3. A monopole source is in the air.

1) Source on the Ground Surface: To model the land-mine detection system in [1], a half space with a dipole source on the ground surface is simulated. The source time function is the Blackman-Harris window function with the center frequency $450 \mathrm{~Hz}$. Fig. 7 shows the four snapshots of the total field on the free ground surface at $2.3 \mathrm{~ms}, 4.0 \mathrm{~ms}, 6.0 \mathrm{~ms}$ and $7.8 \mathrm{~ms}$. The 


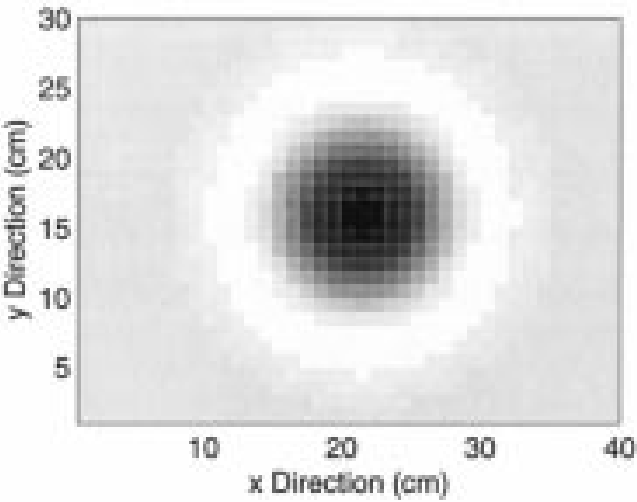

Fig. 11. Energy distribution of the scattered field when the source is in the air above the mine.

primary wave on these snapshots are surface waves. Compared to the surface waves, the $\mathrm{P}$ wave is very weak. The obvious surface wave reflections by the mine are shown on the snapshots at $6.0 \mathrm{~ms}$ and $7.8 \mathrm{~ms}$. The influence of the buried mine on the right-propagating surface wave can be seen clearly on the snapshots. There is a decrease on amplitude when the surface wave passes over the buried mine. Fig. 8(a) shows the waveforms of receiver array 1 . The reflected surface waves are presented on the first five traces. Fig. 8(b) and (c) show the waveforms of receiver arrays 2 and 3 respectively. The decrease of the amplitude can be easily seen on the time traces of receiver 6 to 11 .

2) Source in the Air: Another land-mine detection prototype uses airborne acoustic waves to couple into the ground surface to detect minelike targets [2]. In our simulation, the model consists of two parts: air (pure fluid) and soil (poroelastic medium) with a plastic land mine. A monopole source is in the air $2 \mathrm{~cm}$ above the ground surface. The locations of the receivers are the same as those in the previous model. Fig. 9 shows the four snapshots of vertical velocity field on the ground surface at $2.3 \mathrm{~ms}, 4.0 \mathrm{~ms}$, $6.0 \mathrm{~ms}$ and $7.8 \mathrm{~ms}$. We can see that if a minelike target is present below the surface, the ground vibrational velocity will show distinct changes due to reflection and scattering of surface waves. Fig. 10(a)-(c) show time traces of the scattered field at receiver arrays 1, 2, and 3 respectively. The signature of the buried land mine is clearly present in these figures. It is interesting to note that both direct arrival and the surface waves are scattered by the object into surface waves.

The above results are shown in time domain. However, the prototype in [2] actually displays the acoustic energy in the frequency domain. This display can also be easily done by our time-domain solution. Displayed in Fig. 11 is the energy distribution of the scattered field for a monopole source directly above the mine, showing the presence of the buried object.

\section{CONCLUSION}

A particle velocity-strain, FD method combined with the perfectly matched layer (PML) has been developed for the simulation of acoustic waves propagating in 3-D poroelastic media. The results show the scheme is stable even if the ratio of the fast $\mathrm{P}$ wave velocity to shear wave velocity is greater than 2 .

The difference between elastic model and poroelastic model and moisture effect are investigated by studying surface wave amplitude variation with offset (AVO) in three different ground media: dry sand, fully water saturated sand and partly water saturated sand. The difference between these models is significant. For air saturated sand, the attenuation factor has little influence on AVO. For fully or partly water saturated sand, the attenuation factor greatly affects the AVO response.

This numerical method has been used to investigate the interaction of acoustic waves with a buried mine-like object. Two land-mine detection prototypes are simulated: a half space model with a dipole source on the free ground surface and a half space model with a monopole source in the air. The results show that the wave responses are substantially changed by the presence of the mine-like object, and surface waves play as a primary mechanism of detection at the low frequencies.

\section{REFERENCES}

[1] W. R. Scott Jr., C. T. Schröder, and J. S. Martin, "An hybrid acousto/electromagnetic technique for locating land mines," in Proc. Int. Geoscience and Remote Sensing Symp., Seattle, WA, 1998, pp. 216-218.

[2] J. M. Sabatier and N. Xiang, "Systematic investigation on acoustic-toseismic responses of landmines buried in soil," J. Acoust. Soc. Amer., vol. 107, p. 2896, 1999.

[3] J. Virieux, "SH-wave propagation in heterogeneous media: Velocitystress finite-difference method," Geophysics, vol. 49, pp. 1933-1957, 1986.

[4] Q. H. Liu, F. Daube, C. Randall, E. Schoen, H. Liu, and P. Lee, "A 3D finite difference simulation of sonic logging," J. Acoust. Soc. Amer, vol. 100, pp. 72-79, 1996.

[5] Q. H. Liu and C. Chang, "Compressional head waves in attenuative formations: Forward modeling and inversion," Geophysics, vol. 61, pp. 1908-1920, 1996.

[6] M. A. Biot, "Theory of propagation of elastic waves in a fluid-saturated porous solid. 1. Low-frequency range," J. Acoust. Soc. Amer, vol. 28, pp. $168-178,1956$

[7] - "Theory of propagation of elastic waves in a fluid-saturated porous solid. 1. High-frequency range," J. Acoust. Soc. Amer., vol. 28, pp. 179-191, 1956.

[8] - "Mechanics deformation and acoustic propagation in porous media," J. Appl. Phys., vol. 33, pp. 1482-1498, 1962.

[9] N. Dai, A. Vafidis, and E. R. Kanasewich, "Wave propagation in heterogeneous, porous media: A velocity-stress, finite-difference method," Geophysics, vol. 60, pp. 327-340, 1995.

[10] C. Cerjan, D. Kosloff, R. Kosloff, and M. Reshef, "A nonreflecting boundary condition for discrete acoustic and elastic wave equations," Geophysics, vol. 50, pp. 705-708, 1985.

[11] R. Clayton and B. Engquist, "Absorbing boundary conditions for acoustic and elastic wave equations," Bull. Seismol. Soc. Amer., vol. 67, pp. 1529-1540, 1977.

[12] K. D. Mahrer, "An empirical study of instability and improvement of absorbing boundary conditions for elastic wave equation," Geophysics, vol. 51, pp. 1499-1501, 1986.

[13] J. P. Berenger, "A perfectly matched layer for the absorption of electromagnetic waves," J. Comput. Phys., vol. 114, pp. 185-200, 1994.

[14] W. C. Chew and Q.H. Liu, "Perfectly matched layers for elastodynamics: A new absorbing boundary condition," J. Comput. Acoust., vol. 4, pp. 72-79, 1996.

[15] F. D. Hastings, J. B. Schneider, and S. L. Broschat, "Application of the perfectly matched layer (PML) absorbing boundary condition to elastic wave propagation," J. Acoust. Soc. Amer., vol. 100, pp. 3061-3069, 1996.

[16] Q. H. Liu and J. Tao, "The perfectly matched layer for acoustic waves in absorptive media," J. Acoust. Soc. Amer., vol. 102, pp. 2072-2082, 1997.

[17] Q. H. Liu, "Perfectly matched layers for elastic waves in cylindrical and spherical coordinates," J. Acoust. Soc. Amer., vol. 105, pp. 2075-2084, 1999.

[18] Y. Q. Zeng, J. He, and Q. H. Liu, "The application of the perfectly matched layer in numerical modeling of wave propagation in poroelastic media,” Res. Rep., Duke Univ., Durham, NC, 1999.

[19] R. Burridge and J. B. Keller, "Poroelasticity equations derived from microstructure," J. Acoust. Soc. Amer., vol. 70, pp. 1140-1146, 1981. 
[20] J. Plona, "Observation of the second bulk compressional wave in a porous medium at ultrasonic frequencies," Appl. Phys. Lett., vol. 36, pp. 259-261, 1980

[21] G. Kneib and C. Kerner, "Accurate and efficient seismic modeling in random media," Geophysics, vol. 58, pp. 576-588, 1993.

[22] Q. H. Liu, "Some current trends in numerical methods for transient acoustic and elastic waves in multidimensional inhomogeneous media," Current Topics Acoust. Res., vol. 2, pp. 31-42, 1998.

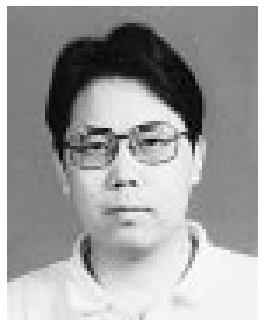

Yan Qing Zeng ( $\left.\mathrm{S}^{\prime} 00\right)$ received the B.S. degree in 1987, the M.S. degree in geophysics in 1990 from Peking University, Beijing, China, and the M.S degree in civil and environmental engineering from Duke University, Durham, NC, in 1999, where he is currently pursuing the Ph.D. degree in electrical and computer engineering.

He was a Faculty Member, Department of Geophysics, Peking University, from 1990 to 1995 . His interests include computational acoustics and electrotion in poroelastic media.
Qing Huo Liu (S'88-M'89-SM'94) received the Ph.D. degree in electrical engineering from the University of Illinois, Urbana, in 1989.

He was with the Electromagnetics Laboratory, University of Illinois, as a Research Assistant from September 1986 to December 1988, and as a Postdoctoral Research Associate from January 1989 to February 1990. He was a Research Scientist and Program Leader with Schlumberger-Doll Research, Ridgefield, CT from 1990 to 1995, and from October 1995 to May 1999, he was with New Mexico State University, Las Cruces. Since June 1999, he has been an Associate Professor of Electrical Engineering with Duke University, Durham, NC. $\mathrm{He}$ has published more than 170 papers in refereed journals and conference proceedings. His research interests include computational electromagnetics and acoustics, wave propagation in inhomogeneous media, geophysical subsurface sensing, and inverse problems.

Dr. Liu is a member of Phi Kappa Phi, Tau Beta Pi, SEC, and a full member of the U.S. National Committee of URSI Commissions B and F. Currently, he serves as an Associate Editor for IEEE TRANSACTIONS ON GEOSCIENCE AND REMOTE SENSING, for which he also served as a Guest Editor for a special issue on computational methods. He received the Presidential Early Career Award for Scientists and Engineers (PECASE) from the National Science and Technology Council, the Early Career Research Award from the Environmental Protection Agency in 1996, and a CAREER Award from the National Science Foundation in 1997. 